\title{
Récepteurs constitutivement actifs et agonisme inverse
}

Les récepteurs membranaires permettent aux cellules eucaryotes de répondre de façon appropriée à de nombreuses stimulations. Cette transduction du signal dépend de la liaison sélective d'un ligand et du changement conformationnel du récepteur induit par cette liaison. Pour les récepteurs couplés aux protéines $G$, ce changement conformationnel permet le couplage fonctionnel du récepteur et de sa protéine $G$ correspondante, entraînant l'activation d'un effecteur intracellulaire. Le couplage entre le récepteur et la protéine $\mathrm{G}$ est donc, normalement, sous le contrôle étroit d'une stimulation adéquate du récepteur. Un certain nombre d'études démontrent, cependant, que des mutations spécifiques de certains récepteurs peuvent abroger ce contrôle et être responsables d'une activation constitutive $\left(\mathrm{m} / \mathrm{s} n^{\circ} 12\right.$, vol. 9, $p$. 1421), [1-3]. Dans ces circonstances, le récepteur, même en absence de son ligand, entraîne l'activation d'une protéine $G$ et de son effecteur. Récemment, de telles mutations ont été associées à des états pathologiques chez l'homme. Une mutation somatique du récepteur de la thyrotropine, qui provoque son activation constitutive, a été détectée chez trois patients sur onze ayant un adénome hypophysaire $\left(\mathrm{m} / \mathrm{s} n^{\circ} 12\right.$, vol. 9, p. 1421) [4]. De même, une mutation causant l'activation constitutive du récepteur de l'hormone lutéinisante $(\mathrm{LH})$ est responsable d'une forme rare de puberté précoce chez les jeunes garçons [5]. Cette maladie se caractérise par une hyperplasie des cellules de Leydig et par une production précoce (dès l'âge de 4 ans) de testostérone. Dans $\mathrm{m} / \mathrm{s} n^{\circ} 1()$, vol. 10 , octobre 94 ces deux cas, il s'agit de récepteurs couplés à Gs qui stimulent la production d'AMPc. Lorsqu'ils sont exprimés dans des cellules COS-7, ces deux récepteurs mutés leur confèrent une augmentation significative de l'activité basale de l'adénylyl cyclase. Dans les cellules thyroïdiennes, cette production accrue de second messager est responsable de la transformation et de la croissance cellulaire incontrôlée qui conduisent à l'apparition d'un adénome, alors que dans les cellules de Leydig elle cause une hyperplasie et une production précoce de testostérone. Une mutation provoquant l'activation constitutive d'un récepteur non hormonal, la rhodopsine, a aussi été impliquée dans une maladie humaine. En effet, la mutation d'une lysine dans le septième passage transmembranaire (Lysine-296) de ce récepteur le rend actif, empêchant la liaison du rétinal, et provoque une dégénérescence progressive de la rétine [6]. De telles mutations, rendant les récepteurs constitutivement actifs, n'entraînent pas nécessairement un dérèglement d'ordre pathologique. Chez la souris, des mutations dans la première boucle cytoplasmique et le deuxième segment transmembranaire du récepteur de la MSH sont associées à l'activation constitutive de ce récepteur qui se traduit par un pelage plus foncé dû à une synthèse accrue de mélanine [7].

Des études de mutagenèse dirigée ont aussi permis de montrer que des mutations ponctuelles peuvent provoquer l'activation constitutive de certains récepteurs. En particulier, Cotecchia et al. ont rapporté que la substitution de trois acides aminés dans la troisième boucle cytoplasmique du récepteur $\alpha_{1 \mathrm{~B}}$-adrénergique lui confère une activité constitutive [1]. La mutation des résidus correspondants dans les récepteurs $\beta_{2}$ - et $\alpha_{2}$ - adrénergiques $[2,3]$ provoque aussi l'activation de ces récepteurs. De façon remarquable, le remplacement de l'alanine en position 293 du récepteur $\alpha_{1 \mathrm{~B}}$-adrénergique par n'importe lequel des 19 autres acides aminés conduit à son activation constitutive [8]. Cette observation que seule une alanine à cette position puisse maintenir le récepteur dans sa forme inactive a amené Lefkowitz [9] à proposer que le récepteur, sous sa forme native, soit dans une conformation contrainte qui empêche l'interaction des domaines d'activation avec la protéine G. Selon ce modèle, l'équilibre entre le conformère inactif (contraint) et le conformère actif (relaxé) serait normalement réglé par la liaison du ligand. Cependant, la mutation de résidus impliqués dans le maintien de la forme contrainte favoriserait le conformère actif du récepteur en relaxant sa structure.

L'hypothèse d'un équilibre dynamique entre les conformères actifs et inactifs est renforcée par une étude récente démontrant que la surexpression du récepteur $\beta_{2}$-adrénergique s'accompagne d'une augmentation de l'activité basale de l'adénylyl cyclase [10]. Ainsi, dans le contexte d'une densité accrue de récepteurs, l'isomérisation spontanée relaxerait une quantité suffisante de récepteur pour permettre la stimulation de Gs et de l'adénylyl cyclase. Cette étude suggère donc qu'une certaine proportion des 
récepteurs natifs peuvent adopter une conformation active, même en l'absence de ligand. Ces travaux ont aussi permis de démontrer que certains antagonistes $\beta$-adrénergiques peuvent inhiber cette propension du récepteur à adopter sa conformation active. Ainsi, des composés tels que le timolol, le propranolol, le betaxolol et l'ICI 118551 possèdent une activité intrinsèque négative permettant l'inhibition de l'activité spontanée du récepteur. Plusieurs de ces agents, nommés agonistes inverses ou antagonistes négatifs, peuvent aussi inhiber l'activité du récepteur $\beta_{2}$-adrénergique rendu constitutivement actif par mutation [11]. C'est vraisemblablement en stabilisant la forme contrainte (inactive) $d u$ récepteur que ces agents exercent leurs effets inhibiteurs. Cependant, les antagonistes $\beta$-adrénergiques ne possèdent pas tous la même " efficacité inverse" vis-à-vis du récepteur spontanément actif. En effet, alors que le timolol peut presque entièrement inhiber l'activité spontanée du récepteur $\beta_{2}$-adrénergique, le labetolol et le pindolol ne sont que de faibles agonistes inverses [10]. Ces derniers peuvent toutefois efficacement bloquer l'action des agonistes et des agonistes inverses de façon compétitive. Il en ressort qu'il existe un spectre d'efficacité inverse allant de l'agoniste inverse complet à l'antagoniste neutre. Les ligands peuvent ainsi être classés comme agonistes, antagonistes neutres ou agonistes inverses selon qu'ils augmentent, laissent inchangée ou diminuent la tendance spontanée du récepteur à adopter une conformation active.

Dans le cas de la rhodopsine, la mutation de la lysine-296 rend le photorécepteur constitutivement actif en empêchant l'attachement du chromophore rétinal. Normalement, c'est l'isomérisation du rétinal en rétinol, à la suite d'une stimulation lumineuse, qui permet l'activation de ce récepteur. On peut donc en déduire que le rétinal maintient le récepteur dans sa conformation inactive et qu'il agit en quelque sorte comme un agoniste inverse endogène. L'existence d'autres ago- inhiber l'activité spontanée de récepteurs hormonaux se présente comme une possibilité intéressante qui mérite d'être explorée. Dans une autre étude récente, Barker $e t$ al. ont montré que la miansérine, un agoniste inverse du récepteur sérotoninergique de type 5-HT2C, et non l'acide 2-bromo-lysergique diéthylamide qui agit comme un antagoniste neutre, provoque une régulation négative du nombre de ce sous-type de récepteur dans le plexus choroïde [12]. Cet eff et de l'agoniste inverse démontre clairement l'importance de l'activité spontanée de ce récepteur dans des conditions physiologiques normales. Pris dans un contexte clinique, ces résultats suggèrent que les agonistes inverses peuvent avoir des effets thérapeuthiques distincts des antagonistes neutres et soulignent l'importance d'une classification rigoureuse.

Les quelques exemples de maladies humaines reliées à une activation constitutive de récepteurs qui ont été présentés ici ne sont certes que les premiers d'une liste qui risque de s'allonger rapidement. L'identification et le développement de composés ayant une bonne activité agoniste inverse permettra sans doute la mise en place de thérapies efficaces pour ces maladies. Mais, en plus de leur intérêt clinique certain, la découverte de nouvelles mutations, de même qu'une meilleure compréhension des mécanismes d'action des agonistes inverses, devraient éclairer d'une lumière nouvelle les processus d'activation et d'inactivation des récepteurs

\section{Michel Bouvier}

Département de biochimie, université de Montréal, C.P. 6128, Succursale CentreVille, Montréal QC H3C 3J7 Canada.

\section{RÉFÉRENCES}

1. Allen LF, Lefkowitz RJ, Caron M, Cotecchia S. G-protein-coupled receptor genes as protooncogenes : constitutively activating mutation of the $\alpha_{18}$-adrenergic receptor enhances mitogenesis and tumorigenecity. Proc Natl Acad Sci USA $1991 ; 88: 11354-8$.

2. Samama P, Cotecchia S, Costa $T$, Lefkowitz RJ. A mutation-induced activated state of the $\beta$,-adrenergic receptor. $J$ Biol Chem 1993 ; 268 : 4625-36.

3. Ren Q, Kurose H, Lefkowitz RJ, Cotecchia S. Constitutively active mutants of the $\alpha_{2}$-adrenergic receptor. I Biol Chem $1993 ; 268: 16483-7$.

4. Parma J, Duprez L, Van Sade J, Cochaux P, Gervy P, Mockel J, Dumont J, Vassart G. Somatic mutations in the thyrotropin receptor gene cause hyperfunctioning thyroid adenomas. Nature $1993 ; 365: 649-51$. 5. Shenker A, Laue L, Kosug S, Merendino J J, Minegishi T, Cutler JB. A constitutively activating mutation of the luteinizing hormone receptor in familial male precocious puberty. Nature $1993 ; 365: 652-4$.

6. Robinson PR, Cohen GB, Zhukovsky E, Oprian DD. Constitutively active mutants of rhodopsin. Neuron $1992 ; 9$ : 719-25.

7. Robbins LS, Nadeau JH, Johnson KR, Kelly MA, Roselli-Rehfus L, Baack E, Mountjoy KG, Cone RD. Pigmentation phenotypes of variant extension locus alleles result from point mutations that alter MSH receptor function. Cell $1993 ; 72: 827-34$.

8. Kjelsberg MA, Cotecchia S, Ostrowski J, Caron M, Lefkowitz RJ. Constitutive activation of the $\alpha_{1 \mathrm{~B}}$-adrenergic receptor by all amino acid substitution at a single site. $J$ Biol Chem 1992 ; 267 : 1430-3.

9. Lefkowitz RJ. Turned on to ill effect. Nature 1993 ; 365 : 603-4.

10. Chidiac $\mathrm{P}$, Hebert $\mathrm{T}$, Valiquette $\mathrm{M}$, Dennis $M$, Bouvier $M$. Inverse agonist activity of $\beta$-adrenergic antagonists. $M o l$ Pharmacol $1994 ; 45: 490-9$.

11. Samama P, Pei G, Costa T, Cotecchia S, Lefkowitz. RJ. Negative antagonists promote an inactive conformation of the $\beta_{2}$. adrenergic receptor. Mol Pharmacol 1994; 45:390-4.

12. Barker EL, Westphal RS, Schmidt D, Sanders-Bush E. Constitutively active 5 . hydroxytryptamine $2 \mathrm{C}$ receptors reveal novel inverse agonist activity of receptor ligands. J Biol Chem 1994 ; 269 : 11687-90.

\section{TIRÉS A PART}

M. Bouvier. 